\title{
Pasireotide for malignant insulinoma
}

\author{
Amit Tirosh,, ${ }^{1,2}$ Salomon M. Stemmer, ${ }^{2,3}$ Evgeny Solomonov, ${ }^{4}$ Eldad Elnekave, ${ }^{3}$ \\ Wolfgang Saeger, ${ }^{5}$ Yelena Ravkin, ${ }^{6}$ Kobi Nir, ${ }^{7}$ Yeela Talmor, ${ }^{8}$ Ilan Shimon ${ }^{1,2}$
}

${ }^{1}$ Endocrine Institute, Rabin Medical Center, Petah Tiqva; ${ }^{2}$ Sackler Faculty of Medicine, Tel Aviv University; ${ }^{3}$ Institute of Oncology, Davidoff Center, Rabin Medical Center, Petah Tiqva; ${ }^{4}$ Surgery, Rivka Ziv medical center, Sefad; ${ }^{5}$ Institute of Neuropathology, University of Hamburg, Germany; ${ }^{6}$ Dan-Petah Tiqva district, Clalit Health Services; ${ }^{7}$ Novartis Pharma Services, Israel; ${ }^{8}$ Internal Medicine F, Rabin Medical Center, Petah Tiqva, Israel

\begin{abstract}
Malignant insulinoma usually has a poor prognosis, as no efficient medical treatment is available. The somatostatin analogs octreotide and lanreotide have limited ability to control the hypoglycemic events. Pasireotide is a multi-receptor targeted somatostatin-analog with improved affinity for SSTR5. There is to date no reported treatment experience with this drug in such tumors. CASE DESCRIPTION: A 72-year-old patient with a G2 stage IV insulinoma, who underwent excision of the primary pancreatic tumor and multiple hepatic metastases, required further treatment for recurrent hypoglycemic events. The glycemic control achieved with pasireotide LAR was better compared with lanreotide and everolimus. However, none of these treatments showed tumor anti-proliferative effects. CONCLUSIONS: Pasireotide monthly injections achieved improved glycemic control in a patient with malignant insulinoma and recurrent hypoglycemic events compared with other medical treatments.
\end{abstract}

Key words: Hypoglycemia, Insulinoma, Neuroendocrine, Pasireotide

\section{CASE REPORT}

We report a 72-year-old male presenting with recurrent fainting episodes. His medical history is unremarkable except for well-controlled hypertension and dyslipidemia. The patient had experienced recurrent fainting episodes, suspected as being syncope, between 2005 and 2010. After the patient complained of severe pre-lunch hunger, blood glucose monitoring

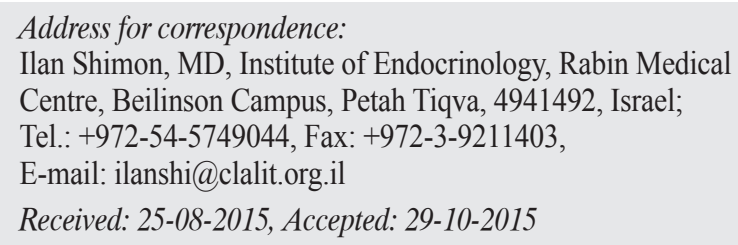

was initiated, revealing recurrent hypoglycemia as low as $60 \mathrm{mg} / \mathrm{dl}(3.3 \mathrm{mmol} / \mathrm{l})$. A subsequent inpatient 72hour fasting test failed to reproduce the hypoglycemia.

In July 2012, the patient fainted at home, at which point his capillary glucose level was $25 \mathrm{mg} / \mathrm{dl}(1.4$ $\mathrm{mmol} / \mathrm{l})$. The patient regained consciousness following a bolus of $50 \%$ dextrose solution and was then hospitalized. C-peptide levels of $3.4 \mathrm{ng} / \mathrm{ml}$ and serum insulin of $14.4 \mathrm{mIU} / \mathrm{ml}$ were observed during an in-house hypoglycemic event (40 mg/dl, 2.2 $\mathrm{mmol} / \mathrm{l})$. The patient and his spouse denied use of insulin secretagogs and no serum anti-insulin antibodies were detected, thus a diagnosis of endogenous hyperinsulinism was made. 
A triple-phase abdominal CT scan demonstrated a $3 \mathrm{~cm}$ lesion in the pancreatic head with multiple hepatic hypervascular masses. CT-guided liver biopsy was consistent with a G2 neuroendocrine tumor (NET), positively stained for chromogranin A and synaptophysin, and 3\% KI-67 expression. The patient underwent a Ga-68 DOTATATE-PET scan, with strong Ga-68 uptake in the pancreatic and liver lesions (Figure 1A).

In November 2012, the patient underwent local excision of the primary tumor from the head of the pancreas, liver segmentectomy (segments 2-3) and multiple wedge excisions of $\sim 10$ liver metastases (Figure 1B). The pathology report was again consistent with a G2 NET (KI-67 of 5\%) positively stained for insulin.
Following surgery, the patient's glucose levels rose and hypoglycemic events diminished, but did not resolve completely. Somatuline autogel $60 \mathrm{mg} /$ month was initiated and increased after one month to $90 \mathrm{mg} / \mathrm{month}$ for several months (Figure 2). However, due to recurrent episodes of hypoglycemia and the patient's complaints of gastrointestinal adverse events, somatuline was discontinued and pasireotide LAR $20 \mathrm{mg}$ was initiated, the dose later being raised to $40 \mathrm{mg}$ monthly. With this treatment regimen the hypoglycemic events almost completely resolved (Figure 2). After several months, new liver metastases were demonstrated on CT scan and the decision was made to switch treatment to everolimus (Affinitor) 10 $\mathrm{mg} /$ day. On this treatment the rate of hypoglycemic events increased (Figure 2) and the patient suffered
A

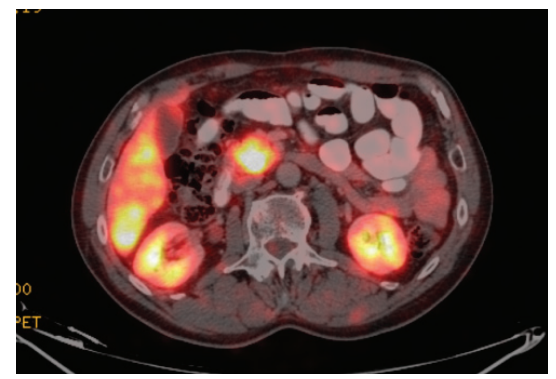

B

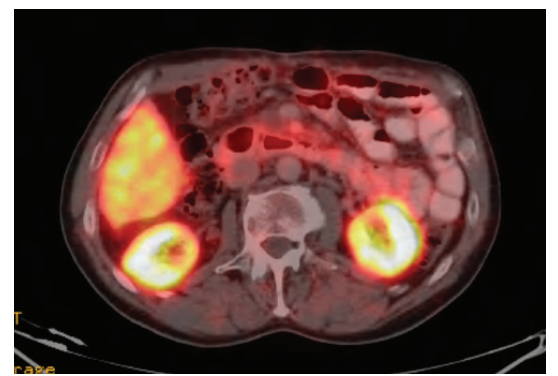

C

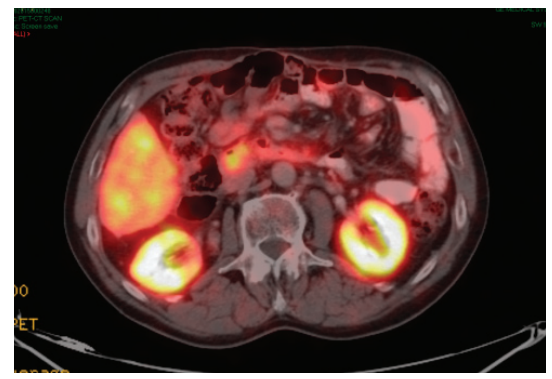

Liver metastases
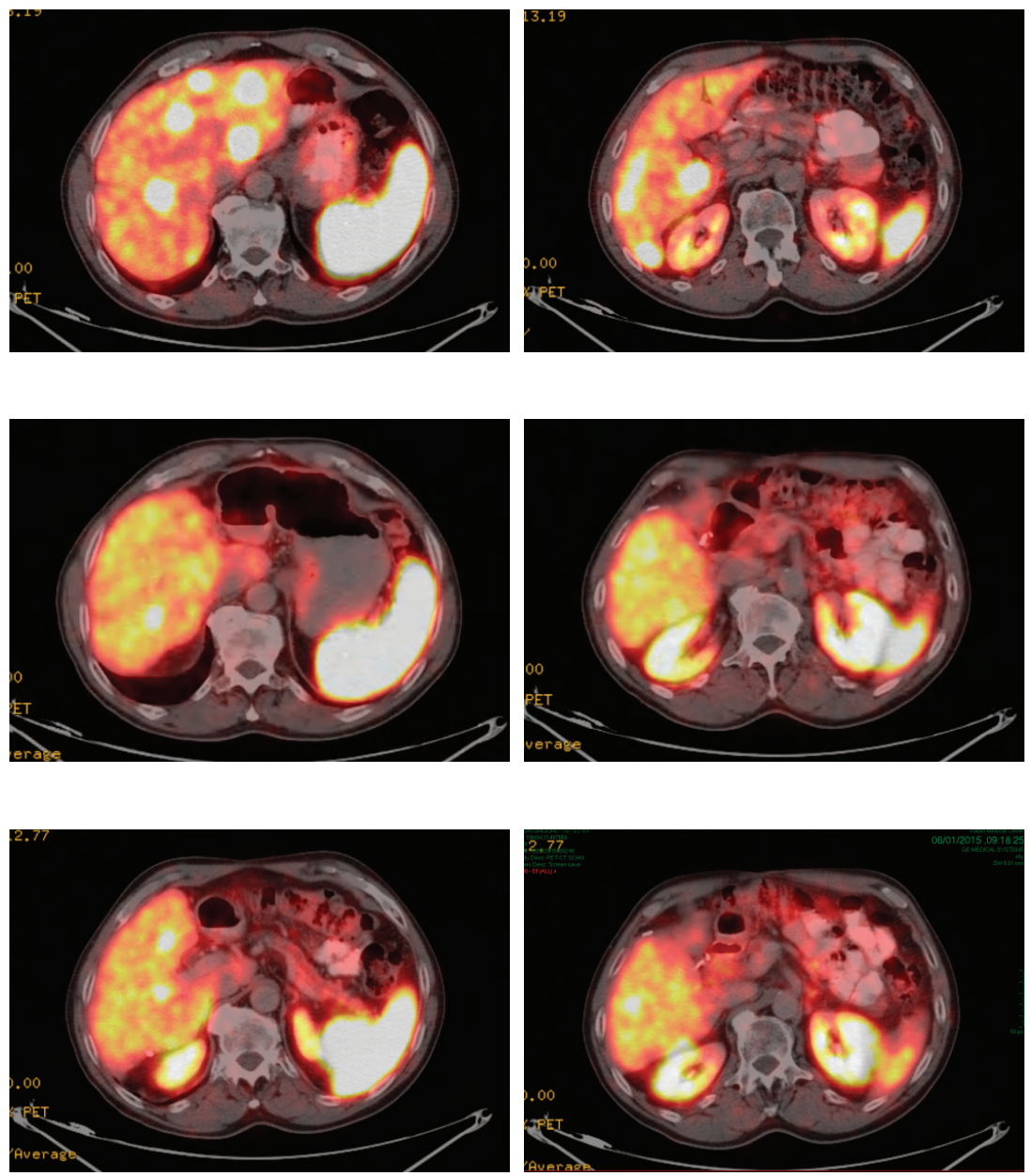

Figure 1. Ga-68 DOTATE-PET scan showing the primary lesion and liver metastases at presentation (A), following surgery (B) and after disease progression (C). White arrow - Primary lesion ( head of the pancreas), green arrows - liver metastases. 

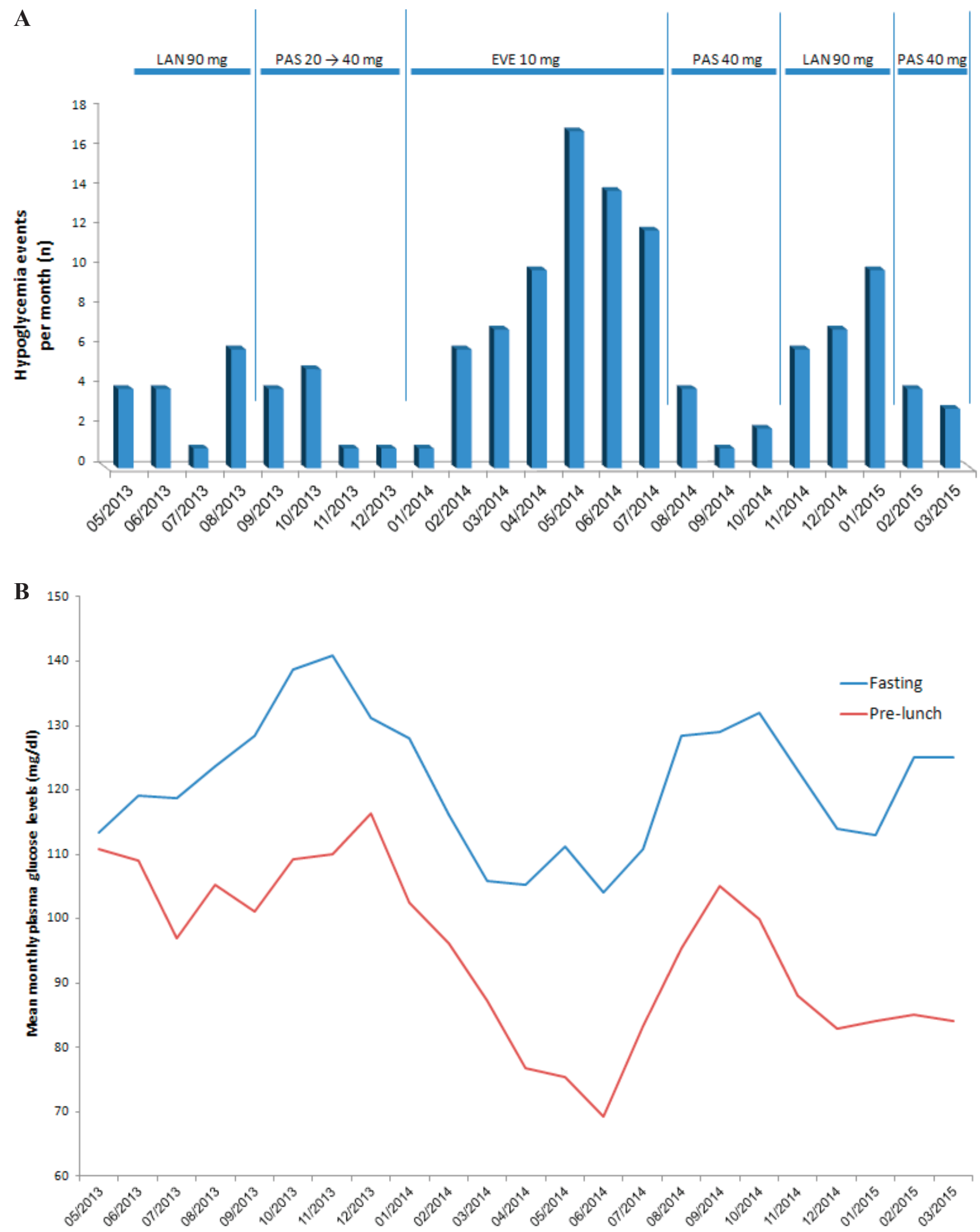

Figure 2. Hypoglycemia (glucose $<70 \mathrm{mg} / \mathrm{dl}$ ) frequency (A), and glucose levels (B), according to fasting and pre-lunch glucose monitoring during follow-up. LAN: lanreotide autogel; PAS: pasireotide LAR; EVE: everolimus. 
from recurrent oral ulcers, fatigue, epistaxis, anemia and moderate neutropenia. Moreover, a new Ga-68 DOTATATE-PET scan showed disease progression (Figure 1C). Thus pasireotide LAR $40 \mathrm{mg} / \mathrm{month}$ was resumed, this resulting in a decrease in hypoglycemic events rate which became rare (Figure 2). Due to temporarily reduced supply of pasireotide at that time, lanreotide was re-administered for several months, leading to worsening glycemic control during this interval. Currently, the patient is being treated again with monthly injections of pasireotide LAR $40 \mathrm{mg}$ with improved control.

In order to study the different effects of somatostatin analogs on plasma glucose levels, we assessed the expression of the different somatostatin receptor subtypes (SSTRs) on the primary tumor and the liver metastases using immunohistochemical staining. We used antibodies against SSTR2a (Zytomed Systems GmbH, Germany), SSTR3 (provided by S. Schulz, Jena, Germany) and SSTR5 (Zytomed). Both the pancreatic lesion and the liver metastases revealed strong membranous staining for SSTR2a and negative for SSTR3 and SSTR5 (Figure 3).

In light of progressive metastatic liver disease, the patient was referred for selective internal radiation therapy (SIRT). Because the overwhelming preponderance of intrahepatic disease was supplied by branches of the right hepatic artery, the patient
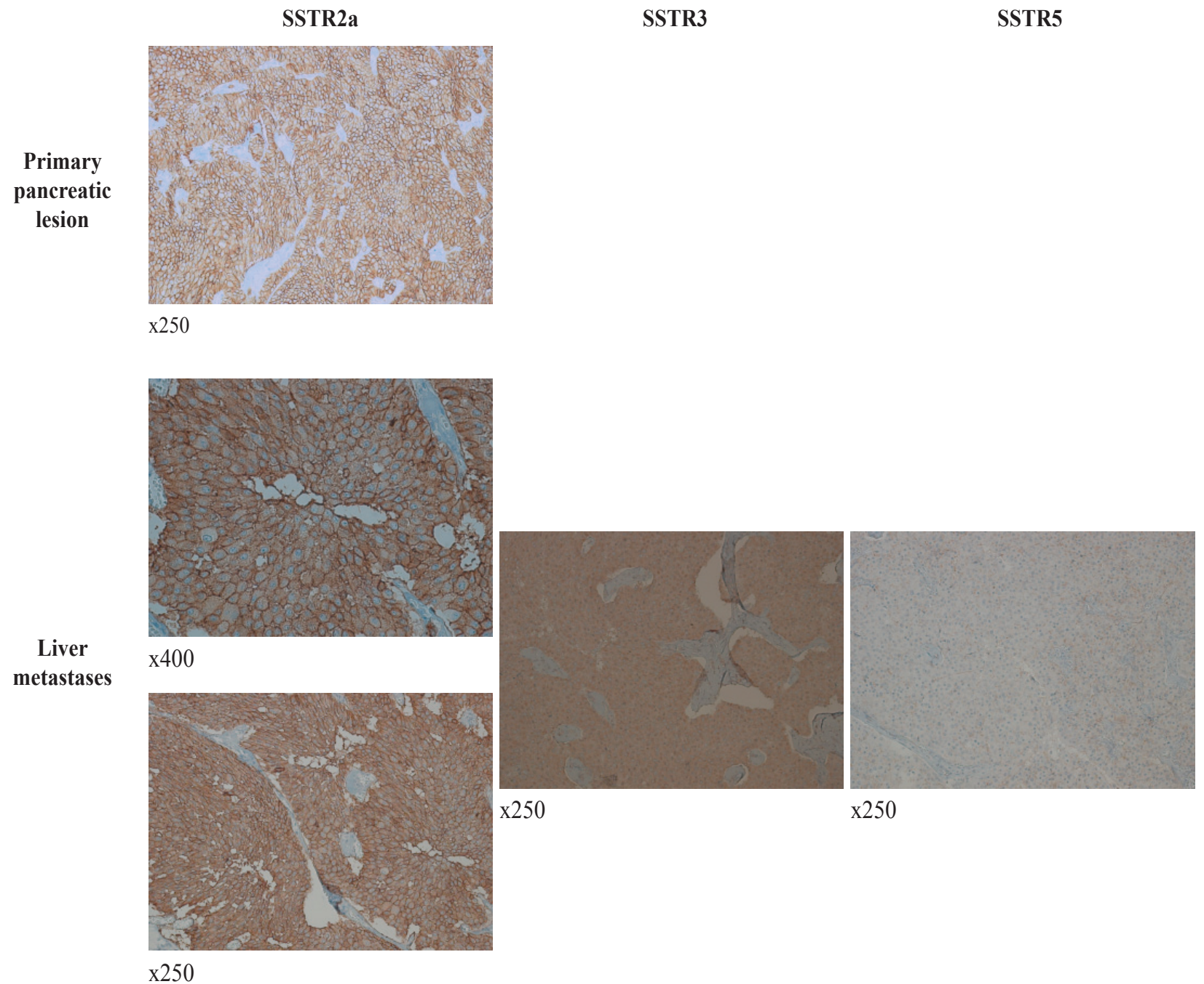

Figure 3. Immunohistochemical staining for SSTRs in malignant insulinoma: positive for SSTR2a and negative for SSTR 3 and SSTR5. SSTR: somatostatin receptor. 
underwent selective right lobar infusion of Yttrium-90 in March 2015, without complications but with mild post-embolization abdominal pain. A follow-up triple phase CT performed 6 months later showed decreased size and near complete non-enhancement of all treated right hepatic lesions.

\section{DISCUSSION}

We describe a patient with a $\mathrm{G} 2$ stage IV insulinoma who underwent excision of the primary tumor and of multiple liver metastases, with temporary reduction in hypoglycemia rate requiring further treatment with somatuline autogel and, ultimately, pasireotide LAR for adequate hypoglycemic control. During the pasireotide treatment period, the patient received everolimus as a second-line therapy due to disease progression, and underwent another treatment period with lanreotide. Better control of hypoglycemia rates was displayed with pasireotide compared with lanreotide or everolimus. However, none of these treatments showed an anti-proliferative effect on this tumor.

Endogenous somatostatin possesses a high affinity for all five known SSTRs. However, synthetic somatostatin analogs have a unique binding affinity profile for each SSTR, which can be an advantage for specific therapeutic purposes. The somatostatin analogs octreotide and lanreotide act mainly through SSTR2 binding. Pasireotide is a multireceptor-targeted second generation somatostatin analog with improved affinity for SSTR1, SSTR2, SSTR 3 and SSTR 5. ${ }^{1}$ The affinity of pasireotide for SSTR5 is 30-40 fold higher, compared with octreotide. ${ }^{1}$ Pasireotide is approved for the treatment of patients with Cushing's disease and acromegaly.

Everolimus inhibits the mammalian target of rapamycin (mTOR) signaling pathway associated with the mTORC1 protein complex, thus exerting an antiproliferative effect. In the RADIANT-3 trial, everolimus prolonged progression-free survival of patients with advanced pancreatic NET ( $24 \%$ were functional) compared with placebo. ${ }^{2}$ Furthermore, patients with hypoglycemic events refractory to treatment with somatostatin analogs showed response to everolimus. ${ }^{3}$

The pronounced effect of pasireotide LAR on hypoglycemic event rate in our patient, compared with the effect of lanreotide (Figure 2), is compatible with the variable expression of the different SSTRs in normal islet cells and insulinomas. Although controversy exists regarding the dominant SSTR responsible for insulin secretion regulation in pancreatic islet cells, ${ }^{4}$ SSTR5 was found to be the main contributor compared to other SSTRs. ${ }^{5}$ In patients with Cushing's disease $^{6}$ and acromegaly ${ }^{7}$ treated with pasireotide, hyperglycemia and diabetes mellitus were common adverse effects. Studies in healthy volunteers suggest that the mechanism of hyperglycemia seen with pasireotide is related to decrease in insulin secretion and significantly decreased incretin response, ${ }^{8}$ resulting from islet cells SSTR5 activation. Importantly, immunohistochemical studies of SSTRs expression in insulinomas found that apart from SSTR2 expression in most (81\%) insulinomas, ${ }^{9}$ SSTR5 was also expressed, but especially in malignant insulinomas. ${ }^{10}$ The immunohistochemical staining demonstrated strong expression of SSTR2 on the pancreatic lesion and the liver metastases, but not SSTR5. The improved glycemic control with pasireotide may thus be explained by the suppression of insulin secretion from normal pancreatic islet cells as seen in patients with Cushing's disease ${ }^{6}$ and acromegaly ${ }^{7}$ treated with pasireotide. The lack of SSTR5 expression on the tumor cells is also in line with the progressive metastatic liver disease recorded despite administration of pasireotide.

In summary, we report the efficacy of different potential medical treatments in reducing hypoglycemic events in a patient with progressive metastatic insulinoma. The improved glycemic control achieved by pasireotide is likely to be related to the pancreatic islet cells SSTR expression profile and to the specific mechanism of action of this molecule.

\section{FUNDING}

The authors declare they have no conflict of interest.

\section{REFERENCES}

1. Bruns C, Lewis I, Briner U, Meno-Tetang G, Weckbecker G, 2002 SOM230: a novel somatostatin peptidomimetic with broad somatotropin release inhibiting factor (SRIF) receptor binding and a unique antisecretory profile. Eur J Endocrinol 146: 707-716. 
2. Yao JC, Shah MH, Ito T, et al, 2011 Everolimus for advanced pancreatic neuroendocrine tumors. N Engl J Med 364: 514-523.

3. Kulke MH, Bergsland EK, Yao JC, 2009 Glycemic control in patients with insulinoma treated with everolimus. N Engl J Med 360: 195-197.

4. Kailey B, van de Bunt M, Cheley S, et al, 2012 SSTR2 is the functionally dominant somatostatin receptor in human pancreatic $\beta$ - and $\alpha$-cells. Am J Physiol Endocrinol Metab 303: E1107-E1116.

5. Zambre Y, Ling Z, Chen MC, et al, 1999 Inhibition of human pancreatic islet insulin release by receptorselective somatostatin analogs directed to somatostatin receptor subtype 5. Biochem Pharmacol 57: 1159-1164.

6. Colao A, Petersenn S, Newell-Price J, et al, 2012 A 12-month phase 3 study of pasireotide in Cushing's disease. N Engl J Med 366: 914-924.
7. Gadelha MR, Bronstein MD, Brue T, et al, 2014 Pasireotide versus continued treatment with octreotide or lanreotide in patients with inadequately controlled acromegaly (PAOLA): a randomised, phase 3 trial. lancet Diabetes Endocrinol 2: 875-884.

8. Henry RR, Ciaraldi TP, Armstrong D, et al, 2013 Hyperglycemia associated with pasireotide: Results from a mechanistic study in healthy volunteers. J Clin Endocrinol Metab 98: 3446-3453.

9. Hofland LJ, Lamberts SWJ, 2003 The pathophysiological consequences of somatostatin receptor internalization and resistance. Endocr Rev 24: 28-47.

10. Portela-Gomes GM, Stridsberg M, Grimelius L, Rorstad O, Janson ET, 2007 Differential expression of the five somatostatin receptor subtypes in human benign and malignant insulinomas - predominance of receptor subtype 4. Endocr Pathol 18: 79-85. 